\title{
Talar Osteochondral Defect Grafting with Nexa Orthopedics OsteoCureтм Bone Graft Plug
}

\author{
by Jonathan Sharpe, DPM ${ }^{1}$, Mark A. Hardy, DPM, FACFAS ${ }^{2}$,
}

The Foot \& Ankle Journal 1 (6): 1

Osteochondritis dessicans of the ankle is a condition often encountered by the foot and ankle physician. Many treatments have been described in the literature including cast immobilization, arthroscopic debridement, open debridement, and autogenous grafting. The NEXA OsteoCure тм bone graft plug allows for immediate lesion excision while avoiding the morbidity associated with obtaining an autograft. The authors provide a brief review of talar dome lesions including staging and classification and their experience and technique involved for utilizing NEXA Orthopedics OsteoCure тм bone graft plugs.

Key words: Osteochondritis dessicans, Talus, Talar dome lesions, NEXA Orthopedics OsteoCureтм bone graft plugs

Accepted: May 2008 Published: June 2008

This is an Open Access article distributed under the terms of the Creative Commons Attribution License. It permits unrestricted use, distribution, and reproduction in any medium, provided the original work is properly cited. @The Foot \& Ankle Journal (www.faoj.org)

$\mathbf{O}_{\mathrm{s}}$ steochondral defects of the talar dome, aka osteochondritis dissecans, are common pathological entities encountered by the podiatric physician. Although trauma is thought to play a primary role in the genesis of these lesions, idiopathic osteonecrosis may also be a cause. Subjectively, these patients frequently present complaining of a deep, aching, non-descript pain in the ankle joint that worsens with activity.

Address correspondence to: Mark A. Hardy, DPM, FACFAS Kaiser Permanente Foundation Department of Podiatric Surgery 12301 Snow Road Parma, OH 44130

Email: markhardy@sbcglobal.net

${ }^{1}$ Senior Resident, Kaiser Permanente/Cleveland Clinic Foundation Residency Program, Cleveland, Ohio.

${ }^{2}$ Director, Cleveland Clinic/Kaiser Permanente. Foot \& Ankle Residency Program. Director, Foot and Ankle Trauma Service. Kaiser Permanente - Ohio Region
Clinical examination may reveal joint line tenderness, effusion, as well as pain upon ankle joint range of motion. Diagnosis is frequently made with imaging after high clinical suspicion, and the lesions are typically seen anterolaterally or posteromedially. (Fig. 1) Insight into the morphology and mechanism of action of these lesions was illustrated in a retrospective study of thirty-one ankles in twenty-nine patients with osteochondral lesions by Canale, et al. ${ }^{1} \quad$ It was found that lateral lesions were associated with inversion or inversion-dorsiflexion trauma and that these lesions are morphologically shallow and anteriorly located on the talar dome. Lateral lesions were more likely to become displaced in the joint and to have persistent symptoms. Medial lesions were both traumatic and atraumatic in origin, morphologically deep, located more posteriorly on the talar dome, and less symptomatic. These typically occurred with a plantarflexion and inversion type of injury. ${ }^{1}$ 


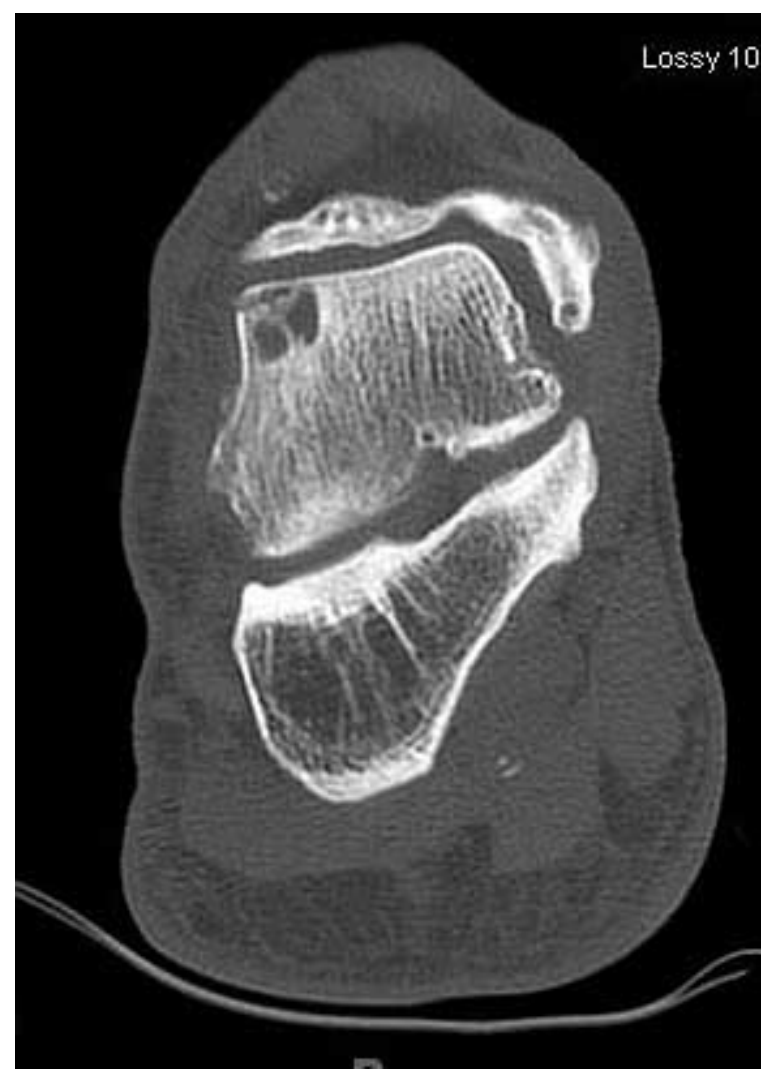

Figure 1 Diagnosis of Osteochondral defects are frequently made with CT scans. The lesions are typically seen anterolaterally or posteromedially.

With an acute injury, the osteochondral lesion may not be visible on the initial radiographs. If there is a high index of suspicion, repeat radiographs in two to four weeks should be obtained or one should consider more advanced imaging. In a study by Anderson, et al., it was found that when plain radiographs of the ankle are relied on for the diagnosis of an osteochondral fracture of the talus, many lesions remain undiagnosed. ${ }^{2}$ Stage-I osteochondral fractures show no diagnostic changes on plain radiographs, and Stage-II lesions are usually subtle and, therefore, are often overlooked by both radiologists and clinicians. The most commonly used classification system for these injuries was created by Berndt and Harty. ${ }^{3}$

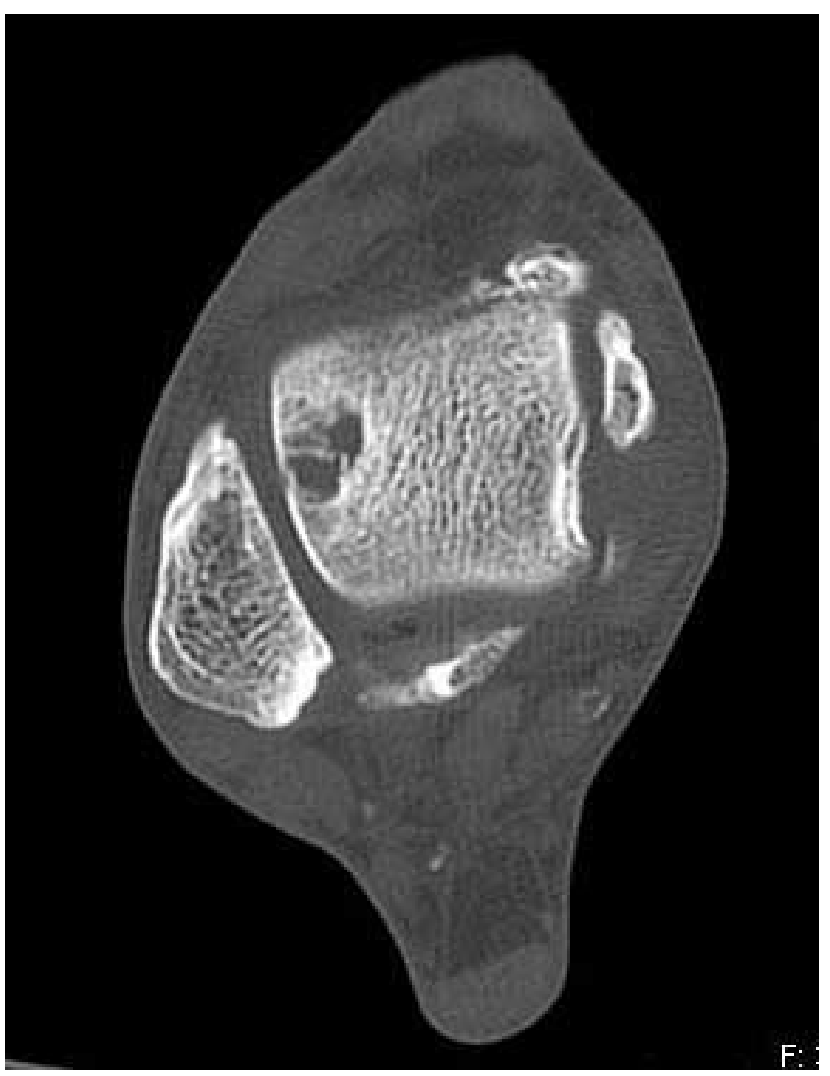

Figure 2 A CT may offer more accurate staging of the lesion, although classification may not correlate with intraoperative findings.

A type I lesion represents a small area of compression. A type II lesion is a partially detached osteochondral lesion. When the lesion becomes completely detached, but remains in its anatomical location, it is a type III lesion. A detached lesion with any movement or migration is classified as type IV. A CT may offer more accurate staging of the lesion, although classification may not correlate with intraoperative findings. (Fig. 2) Pettine, et al., evaluated seventyone osteochondral fractures of the talus for an average of 7.5 years after the onset of symptoms to determine which factors influenced the final result. It was found that the type of fracture was the most important factor and that delay in treatment also affected the results adversely. ${ }^{4}$ 
In the study by Canale, et al., using the classification system of Berndt and Harty, it appeared that Stage-I and Stage-II lesions should be treated non-operatively, regardless of location. Stage-III medial lesions should be treated nonoperatively initially but if symptoms persist, surgical excision and curettage are indicated. Stage-III lateral lesions and all Stage-IV lesions should be treated surgically and early. Long-term results indicated that few lesions unite when treated non-operatively. Degenerative changes in the ankle joint, whether symptomatic or not, were common regardless of the type of treatment. ${ }^{1}$

Non-operative treatment of these lesions includes casting and immobilization. There is no evidence, however, that these patients need to be immobilized if they are kept non-weight bearing. There is also no evidence that a non-weight bearing cast offers better results than a weight bearing cast.

A retrospective study of 22 ankles in 22 patients with osteochondral talar dome lesions between 1975 and 1983 indicated that surgical treatment yields superior results to conservative therapy. ${ }^{5}$ Many of these lesions are treated surgically with arthroscopic joint examination and debridement of the lesion. This process may be aided by an external joint distracting device. Anterolateral lesions are typically more amenable to arthroscopic debridement than posteromedial lesions because of their anatomical location. In a study by Kumai, et al., the authors found good clinical results in arthroscopic debridement and $\mathrm{k}$ wire drilling of lesions in patients who were younger than sixty years old. ${ }^{6}$ Posteromedial lesions typically necessitate an osteotomy of the medial malleolus for exposure, with open reduction and internal fixation and subsequent prolonged non weight-bearing. (Fig. 3)

We have employed the NEXA Orthopedics OsteoCureтм Bone Graft Plug with success for surgical repair of talar osteochondral lesions. The OsteoCureтм Plug is a cylindrical implant ideal for filling defects in bone.

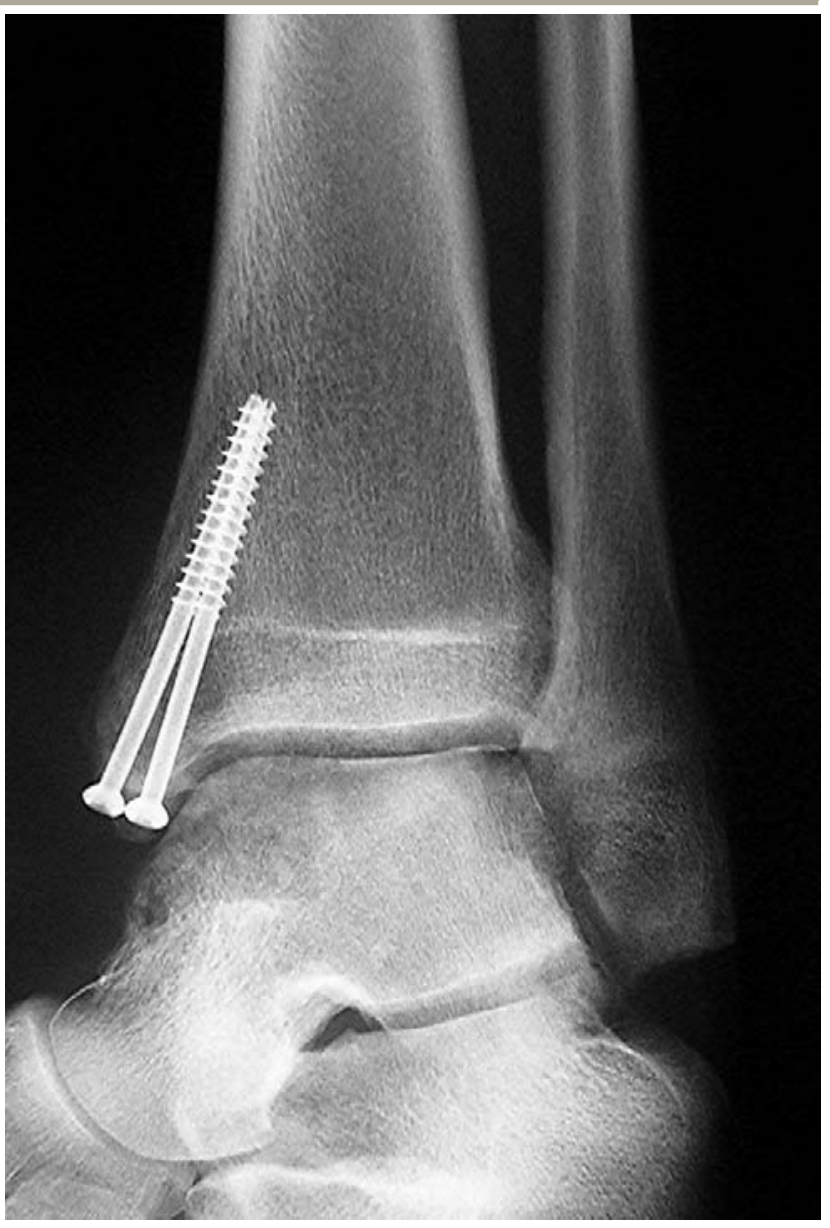

Figure 3 Posteromedial lesions typically necessitate an osteotomy of the medial malleolus for exposure. Here, ORIF screws are placed through the medial malleolus following the procedure.

The implant is a resorbable, porous scaffold, which allows the in-growth of new healing tissue. The implant is sized for cylindrical defects with a minimum depth of $5 \mathrm{~mm}$ and a maximum depth of $12 \mathrm{~mm}$. Careful pre-operative planning should be taken to ensure adequate visualization of the defect to be grafted. An osteotomy may need to be employed to ensure sufficient access to the lesion. 


\section{Surgical Technique}

The following described technique has been outlined from NEXA's surgical technique literature. The OsteoCure ${ }^{\mathrm{TM}}$ Plug Implant Kit contains the cylindrical implant, the delivery device, and a trimming knife. This is to be used with the accompanying OsteoCure ${ }^{\mathrm{TM}}$ Plus Site Preparation Kit. (Fig. 4) The standard implant depths range from $5 \mathrm{~mm}$ to $12 \mathrm{~mm}$, but longer implants are available. The implants also come in a variety of diameters to suit the varying sizes of these lesions. The color-coded instrument set comes in 5, 7, 9, and $11 \mathrm{~mm}$ to correspond to the accompanying OsteoCure ${ }^{\mathrm{TM}}$ implant sizes. Once adequate visualization and access of the talar lesion has been obtained, a thin walled drill sleeve is introduced to the talar dome, taking care to encompass the entire lesion. (Figs. 5-7)

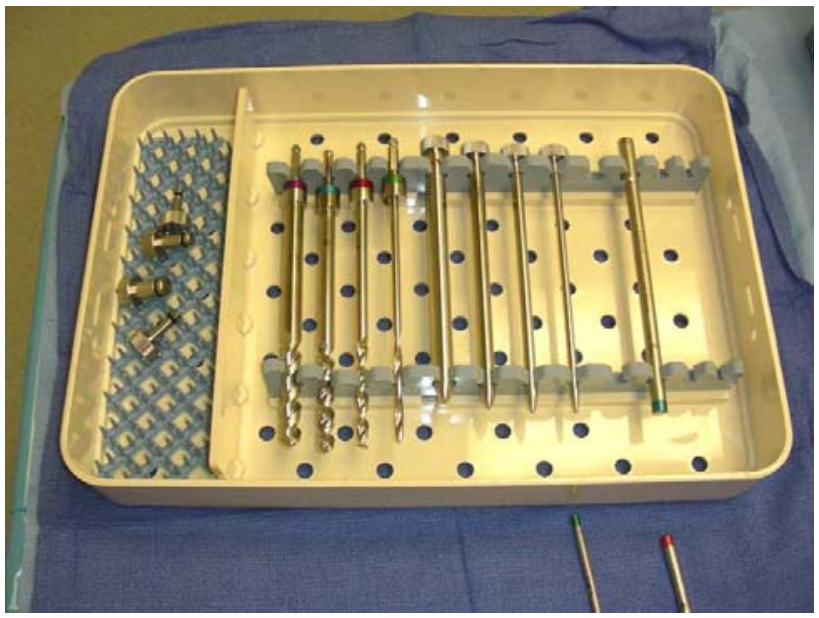

Figure 4 The OsteoCure ${ }^{T M}$ Plug Implant kit contains the cylindrical implant, the delivery device, and a trimming knife. This is to be used with the accompanying OsteoCure ${ }^{\mathrm{TM}}$ Plus Site Preparation Kit.
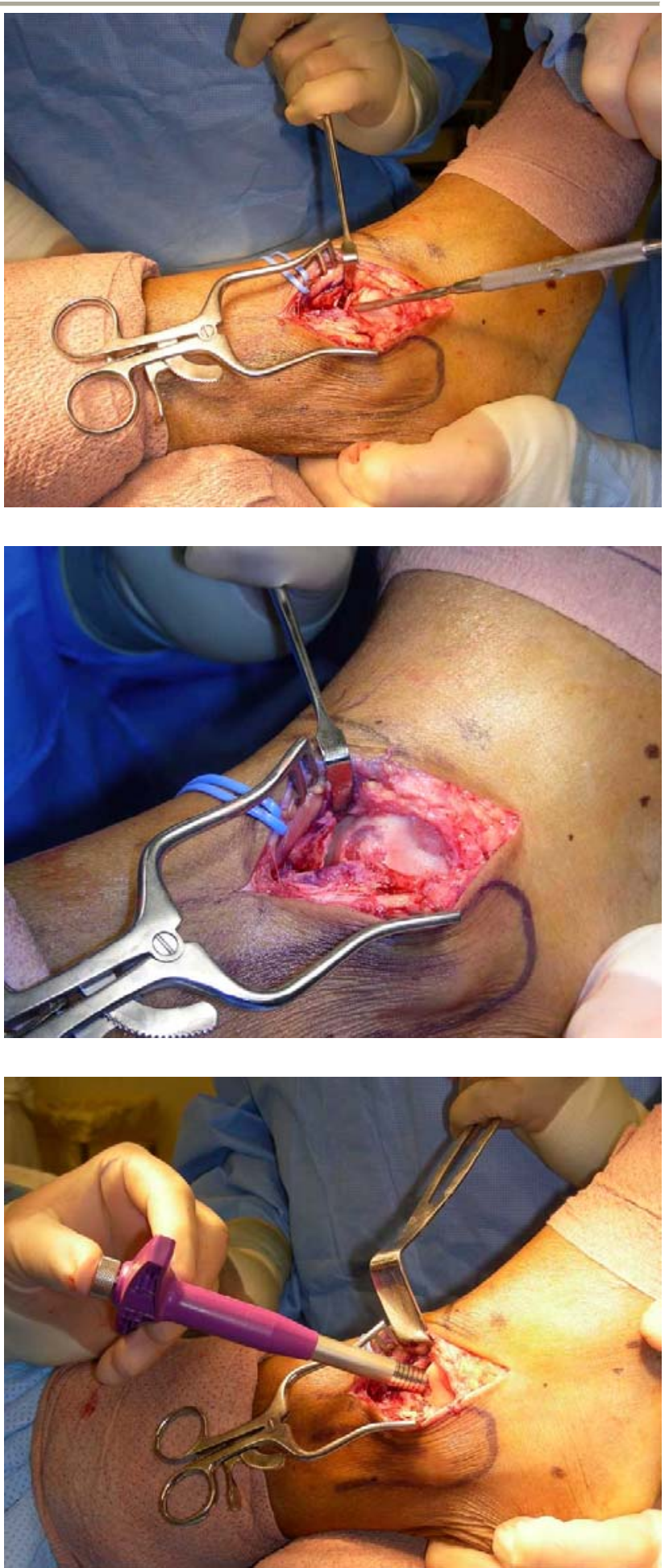

Figures $\mathbf{5 , 6 , 7}$ Once the osteochondral defect is identified, a thin walled sleeve drill is introduced to the talar dome. 


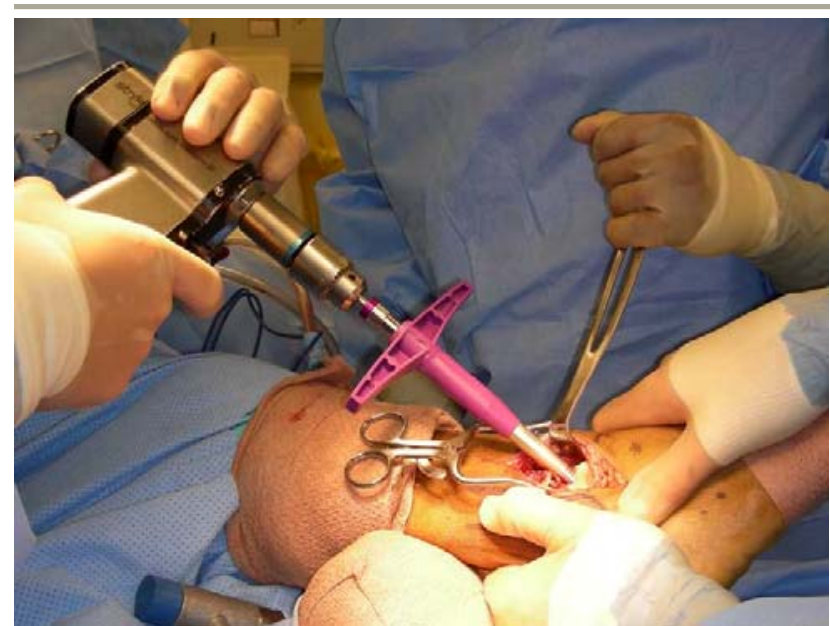

Figure 8 Care is taken to insert the drill sleeve and drill in a manner perpendicular to the articular surface to ensure that the graft will lie flush after insertion.

Prior to insertion, an appropriately sized obturator is introduced into the handle of the drill sleeve for ease of insertion. The drill sleeve is then gently introduced through the cartilaginous surface by pronating and supinating the device. Once the drill sleeve has been slightly advanced, remove the obturator from the drill sleeve. The depth can easily be measured on the drill sleeve, which is calibrated in millimeter measurements. Care must be taken to insert the drill sleeve in a manner perpendicular to the articular surface to ensure that the graft lies flush after insertion. Next, the metallic cap is removed while maintaining the drill sleeve at the desired depth. The corresponding drill is then introduced into the drill sleeve and the lesion is drilled to the desired depth. (Fig. 8) Continue drilling until the drill stop contacts the drill sleeve. The drill and drill sleeve are then both removed from the surgical field. (Fig. 9) Next, the cylindrical graft is prepared. The graft comes housed in a delivery device.

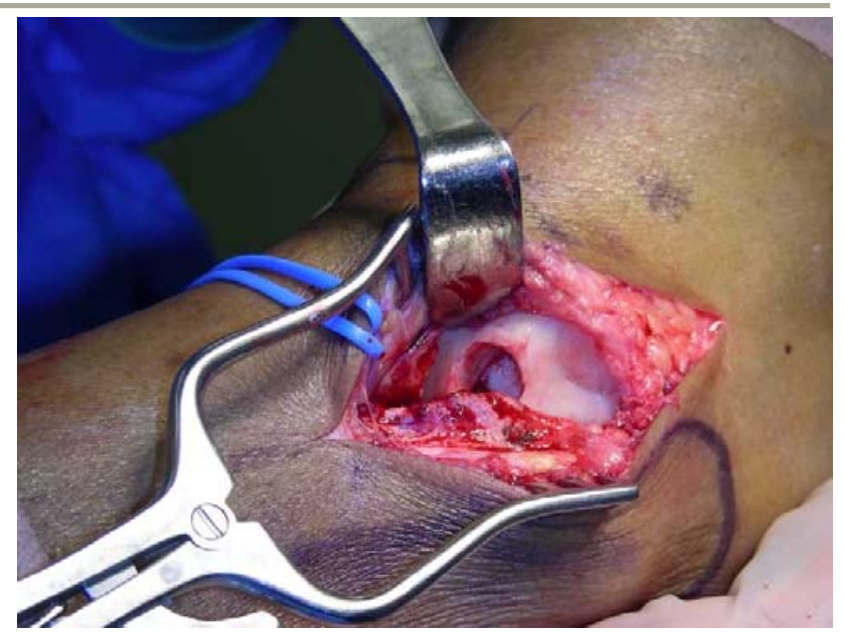

Figure 9 The talar dome defect is now drilled and prepared for grafting.

The device has a plunger on the opposite end of the graft. The plunger end should be introduced into the lesion and pressed firmly into place. This will force the graft out of the proximal end of the delivery device, effectively sizing the implant. (Fig. 10)

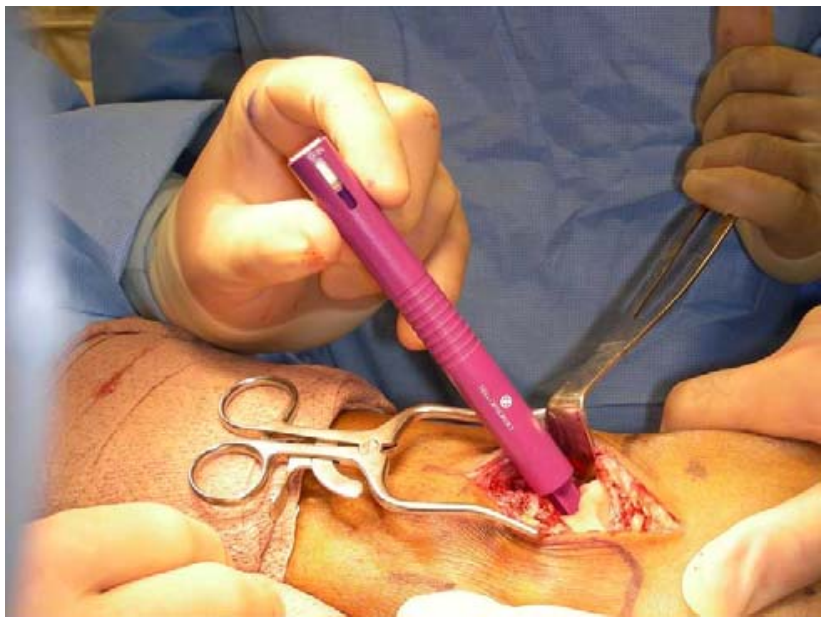

Figure 10 The plunger end of the device is introduced, effectively sizing the implant. 

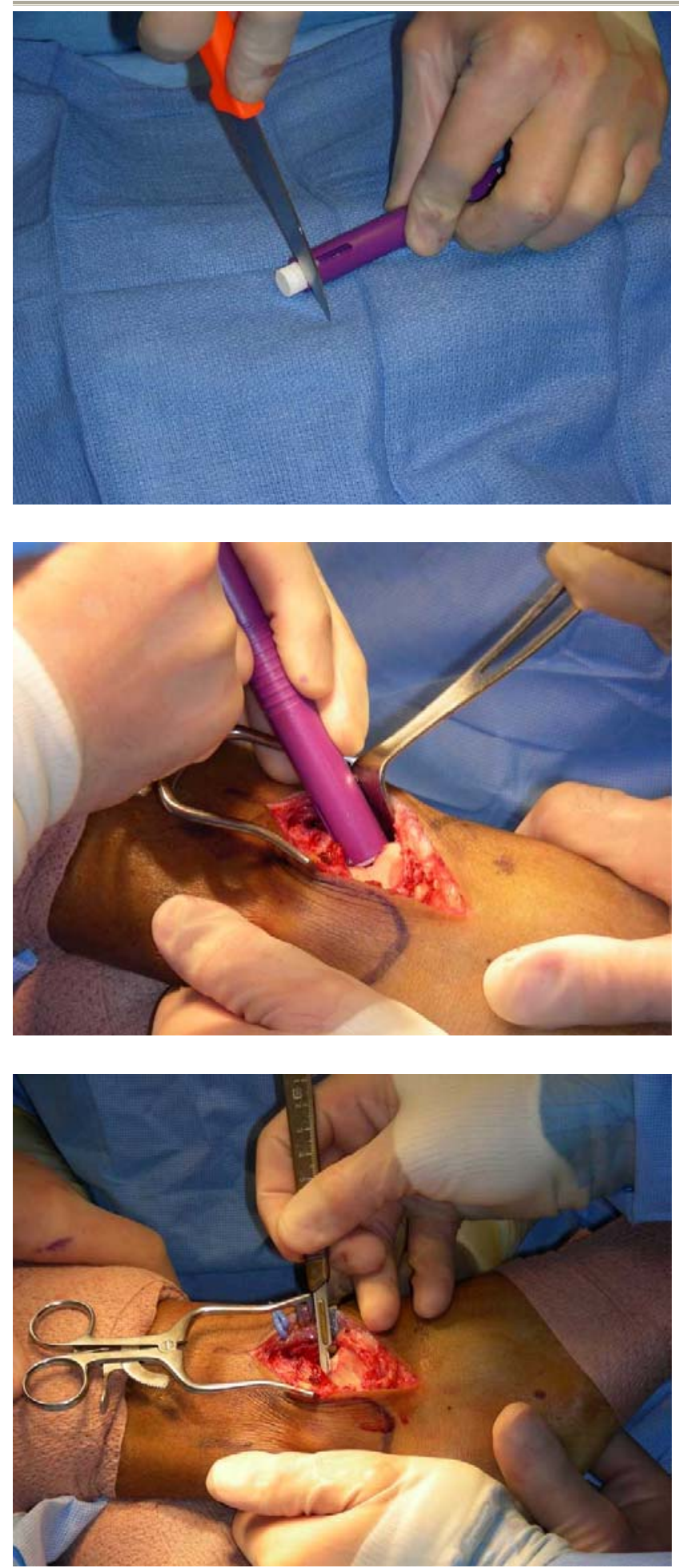

Figures 11,12,13 The redundant portion of graft is trimmed. (Fig. 11) The graft is introduced (Fig. 12) and gently tamped into place. Gently contour the graft as needed with a scalpel. (Fig. 13)

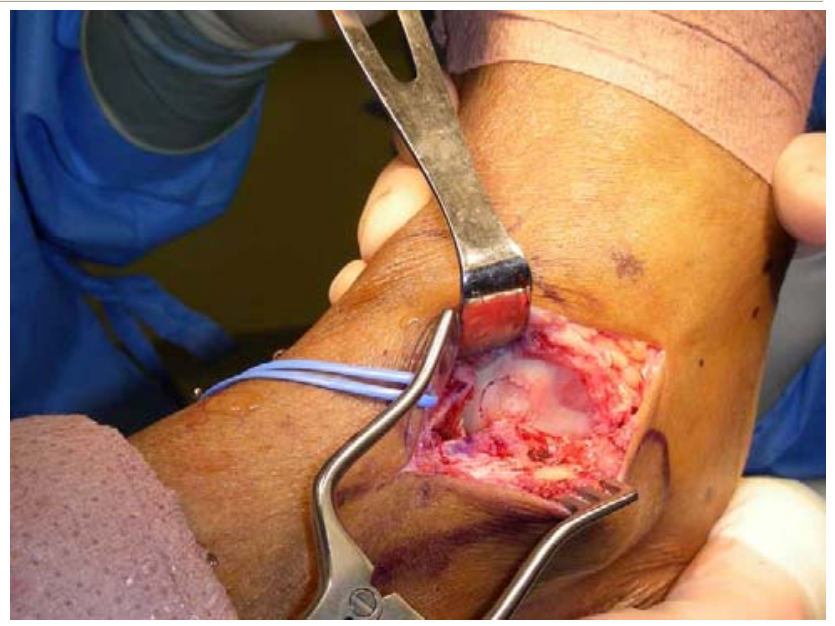

Figure 14 The graft is seated in place. ORIF of the malleolus is then performed with appropriate closure of the surgical site.

The delivery device is then removed from the field and the redundant overhanging graft is trimmed with the knife included in the kit. (Fig. 11) The delivery device is then reintroduced to the lesion with the graft now facing distally toward the lesion. It is helpful to advance the graft a few millimeters to help introduce it into the drilled defect. The plunger on the proximal end of the delivery device is then firmly advanced, seating the graft into the defect. (Fig. 12) It can be gently tamped with a mallet. The graft should sit flush with the articular surface. If there is a small step-off deformity, gently contour the graft with a scalpel. (Fig. 13) Once the graft has been placed, commence with ORIF of osteotomy if necessary and standard wound closure. (Fig. 14) 
According to NEXA, immediately following implantation the scaffold allows blood, marrow and progenitor cells to be transported into the pores. The scaffold provides a porous and mechanically protected environment for healing, tissue in-growth and cellular development. The calcium sulfate dissolves within 6 weeks to 6 months. New tissue forms within the pores. Between 6 months and 12 months, the polymer undergoes resorbtion and is gradually replaced with tissue as well. After 12 months the polymer is predominantly resorbed and the defect will contain new tissue.

Our post-operative treatment protocol consists of non-weight bearing for 2 weeks, followed by active range-of-motion, usually, once the incisions have healed. Partial weight bearing is then employed for 2 weeks, in a fracture boot, with progression to full weight bearing for an additional 2 weeks.
At 6-8 weeks the patient is then enrolled into a formal physical therapy program.

\section{References}

1. Canale-ST; Belding-RH Osteochondral lesions of the talus JBJS-Am. 62(1): 97-102, Jan. 1980.

2. Anderson IF, et al: Osteochondral fractures of the dome of the talus JBJS - Am. 71(8):1143-52, Sept. 1989.

3. Brendt, AL, Harty, M. Transchondral fractures (osteochondritis dissecans) of the talus. JBJS. Vol 41-A. 988-1020, 1959.

4. Pettine KA. Morrey BF. Osteochondral fractures of the talus. A long-term follow-up. JBJS - British 69(1):89-92, Jan. 1987.

5. Flick AB. Gould N. Foot \& Ankle. Osteochondritis dissecans of the talus (transchondral fractures of the talus): review of the literature and new surgical approach for medial dome lesions. [JC:f3x] 5(4):165-85, Jan-Feb 1985. 6. Kumai T, Takakura Y, Higashiyama I, Tamai S. Arthroscopic drilling for the treatment of osteochondral lesions of the talus. J Bone Joint Surg Am. 81(9):1229-35, Sept, 1999. 